\title{
Non-muscle myosin II knockdown improves survival and therapeutic effects of implanted bone marrow-derived mesenchymal stem cells in lipopolysaccharide-induced acute lung injury
}

\author{
Guosheng Wu ${ }^{1 \#}$, Fei Chang ${ }^{2 \#}$, He Fang ${ }^{1 \#}$, Xingfeng Zheng ${ }^{1 \#}$, Mingzhu Zhuang ${ }^{3 \#}$, Xiaobin Liu ${ }^{1}$, \\ Wenjia Hou ${ }^{1}$, Long $\mathrm{Xu}^{1}$, Zhengli Chen ${ }^{1}$, Chenqi Tang ${ }^{1}$, Yu Wu ${ }^{4}$, Yu Sun ${ }^{1}$, Feng Zhu ${ }^{1}$ \\ ${ }^{1}$ Department of Burn Surgery, Changhai Hospital, Naval Medical University, Shanghai, China; ${ }^{2}$ Department of Burn and Plastic Surgery, The \\ Affiliated Zhang Jiagang Hospital of Soochow University, Suzhou, China; ${ }^{3}$ Clinical BioBank, Changhai Hospital, Naval Medical University, \\ Shanghai, China; ${ }^{4}$ Department of Anesthesiology, Bethune International Peace Hospital, Shijiazhuang, China \\ Contributions: (I) Conception and design: G Wu, F Chang, H Fang, X Zheng, M Zhuang, Y Sun, F Zhu; (II) Administrative support: None; (III) \\ Provision of study materials or patients: None; (IV) Collection and assembly of data: X Liu, W Hou, L Xu, Z Chen, C Tang, Y Wu; (V) Data analysis \\ and interpretation: G Wu, F Chang, H Fang, X Zheng, M Zhuang, Y Sun, F Zhu; (VI) Manuscript writing: All authors; (VII) Final approval of \\ manuscript: All authors. \\ "These authors contributed equally to this work. \\ Correspondence to: Dr. Feng Zhu, MD, Professor; Dr. Yu Sun, MD, Professor. Department of Burn Surgery, Changhai Hospital, Naval Medical \\ University, Shanghai 200433, China. Email: alexzhujunchi@hotmail.com; littlefish0916@126.com.
}

Background: Bone marrow-derived mesenchymal stem cells (BMSCs) have been shown to have some beneficial effects in acute lung injury (ALI), but the therapeutic effects are limited due to apoptosis or necrosis after transplantation into injured lungs. Here, we aim to explore whether Non-muscle myosin II (NM-II) knockdown could enhance BMSCs survival and improve therapeutic effects in ALI.

Methods: MSCs, isolated from rat bone marrow, were transfected with the small interfering RNA (siRNA) targeted to NM-II mRNA by a lentivirus vector. Rats were equally randomized to four groups: the control group was given normal saline via tail vein; the other three groups underwent intratracheal lipopolysaccharide (LPS) instillation followed by administration with either normal saline, BMSCs transduced with lentivirusenhanced green fluorescent protein (eGFP) empty vector, or BMSCs transduced with lentivirus-eGFP NMII siRNA. Hematoxylin and eosin staining was used to evaluate lung histopathologic changes and Masson trichrome staining was used to assess lung fibrosis. The myeloperoxidase activity was also tested in lung tissues. The mRNA expression of inflammatory cytokines in lung tissues was determined via quantitative reverse transcription PCR. Sex-determining region of the Y chromosome gene expression was measured by fluorescence in situ hybridization (FISH) assay. The expression of self-renewal activity and apoptosisassociated proteins were measured by Western blot.

Results: Transplantation of NM-II siRNA-modified BMSCs could improve histopathological morphology, decrease inflammatory infiltrates, down-regulate the expression levels of inflammatory cytokines, and reduce pulmonary interstitial edema. NM-II siRNA-modified BMSCs showed antifibrotic properties and alleviated the degrees of pulmonary fibrosis induced by endotoxin. In addition, NM-II knockdown BMSCs showed slightly better therapeutic effect on lung inflammation when compared with control BMSCs. The beneficial effects of NM-II siRNA-modified BMSCs may be attributed to enhanced self-renewal activity and decreased apoptosis.

Conclusions: NM-II knockdown could inhibit the apoptosis of implanted BMSCs in lung tissues and improve its self-renewal activity. NM-II siRNA-modified BMSCs have a slightly enhanced ability to attenuate lung injury after LPS challenge. 
Keywords: Bone marrow-derived mesenchymal stem cells (BMSCs); acute lung injury (ALI); non-muscle myosin II (NM-II); gene therapy

Submitted Jun 21, 2020. Accepted for publication Nov 02, 2020.

doi: $10.21037 /$ atm-20-4851

View this article at: http://dx.doi.org/10.21037/atm-20-4851

\section{Introduction}

Mesenchymal stem cells (MSCs), an ideal cell type for cellbased therapies, have been shown to have beneficial effects in several lung diseases, including sepsis-induced acute lung injury (ALI), ischemia and reperfusion-induced ALI, white smoke inhalation injury, chronic obstructive pulmonary disease (COPD), and lung fibrosis (1-4). Bone marrowderived MSCs (BMSCs) are one of the several types of stem cells. Our previous work, combined with other authors' findings, has revealed that intravenously administered exogenous BMSCs mainly home to lung tissues, where they repair injured lung tissues by differentiating into mature lung cell types or secreting various factors involved in tissue repair $(5,6)$. Several clinical trials investigating MSCs for acute respiratory distress syndrome are in progress, and some have revealed no safety issues in phase $1 \mathrm{~b}$ trials (7-9). However, the low survival rates of implanted BMSCs in damaged tissues due to apoptosis or necrosis hinders their therapeutic effects $(10,11)$.

Non-muscle myosin II (NM-II), an actin-binding protein with actin cross-linking and contractile properties, participates in several essential cellular processes, including mitosis, migration, adhesion, differentiation, and polarization (12). Studies have demonstrated that NM-II inhibitors can promote self-renewal or differentiation of pluripotent stem cells into specific cell types (13). Here, we aimed to investigate whether NM-II knockdown could inhibit the apoptosis of implanted BMSCs in lung tissues, thereby increasing cloning efficiency and improving the therapeutic effects of BMSCs. We present the following article in accordance with the ARRIVE reporting checklist (available at http://dx.doi.org/10.21037/atm-20-4851).

\section{Methods}

\section{Reagents}

Lipopolysaccharide (LPS, Escherichia coli 0111:B4) was obtained from Sigma-Aldrich (St. Louis., MO, USA). Cell culture reagents were purchased from KeyGen Biotech
(Nanjing, China). Lipofectamine ${ }^{\circledR} 3000$ was procured from Invitrogen (Carlsbad, CA, USA). Opti-MEM ${ }^{\circledR}$ reducedserum medium was purchased from Thermo Fisher Scientific Inc. (Waltham, MA, USA). Anti-CD34, CD44, CD45, CD29, and CD90 antibodies for flow cytometry were purchased from Abcam (Cambridge, MA, USA). Rabbit polyclonal antibodies against Angiopoietin-1 (Ang-1), RhoA-associated kinase 1 (ROCK1) and ROCK2 were obtained from Abcam Inc. (Cambridge, MA, USA). Rabbit polyclonal antibodies against NM-II and Caspase-3 were provided by Bioss Inc. (Woburn, MA, USA). The rabbit polyclonal anti-Nanog antibody was purchased from Affinity Biosciences Inc. (Cincinnati, OH, USA) and a mouse monoclonal anti-GAPDH antibody was from ZSGB Bio (Beijing, China). Horseradish peroxidase-conjugated secondary antibodies were provided by Santa Cruz Biotechnology (Dallas, Texas, USA). A myeloperoxidase (MPO) assay kit was purchased from Bioengineering Institute (Nanjing, China). An in situ hybridization detection kit for the SRY (sex-determining region of the Y chromosome) gene was purchased from BosterBio (Wuhan, China). Enzyme linked immunosorbent assay (ELISA) kits for Interleukin 1 (IL-1), IL-6 and tumor necrosis factor- $\alpha$ (TNF- $\alpha$ ) were obtained from R\&D Systems (Minneapolis, $\mathrm{MN}, \mathrm{USA})$.

\section{Isolation and culture of Rat BMSCs}

Male Sprague-Dawley (SD) rats, purchased from the Experimental Animal Center, Naval Medical University (Shanghai, China) were housed in an animal care system (ACS) with a $12 \mathrm{~h} \mathrm{light/dark} \mathrm{cycle.} \mathrm{This} \mathrm{work} \mathrm{was} \mathrm{approved}$ by the Animal Care and Use Committee of the Naval Medical University (Approval ID: SYXK(HU)2017-0004), in compliance with guide for care and use of laboratory animals published by the U.S. National Institutes of Health (NIH) (publication No. 96-01). MSCs were isolated from the bone marrow of femur and tibia bones of rats as previously reported (14). Briefly, a 21-gauge needle was used to flush the bone marrow cavity with $30 \mathrm{~mL}$ 
complete medium containing 20\% fetal bovine serum (FBS), L-glutamine, penicillin, streptomycin, and amphotericin. Harvested cells were filtered and cultured in a $10 \mathrm{~cm}$ dish. After growing to $90 \%$ confluence, the adherent cells were harvested and cryopreserved. Flow cytometry was used to identify the stem-like characteristics of the isolated cells. Cell surface markers CD34, CD44, CD45, CD29, and CD90 were detected. BMSCs at passage 3 were used in the following in vivo and in vitro experiments.

\section{Lentivirus transduction of BMSCS}

Small interfering RNA (siRNA) targeting NM-II mRNA was synthesized by General Biosystems Co. Ltd. (Anhui, China). The DNA target sequence for NM-II-targeted siRNA was 5'-GUGUGGUCAUUAAUCCUUA-3', and the scrambled control RNA was 5'-UUCUCCGAACGUGUCACGU-3'. Lentiviral transduction of BMSCs was performed in accordance with the manufacturer's instructions. In brief, cells were grown to the logarithmic phase, harvested, and washed three times with phosphate-buffered saline (PBS). Cells were re-suspended $\left(2.5 \times 10^{5} /\right.$ well $)$ in $0.5 \mathrm{~mL}$ of complete medium (RPMI 1640 with $10 \%$ FBS) in 12 well plates. Lentivirus was added to a multiplicity of infection (MOI) of 35 colony-forming units per cell. Cells were then incubated for $12 \mathrm{~h}$ before washing and adding fresh medium. Cells were subsequently transferred to 6-well plates and $10 \mathrm{~cm}^{2}$ bottles, and siRNA-expressing cells were selected in puromycin $(5 \mu \mathrm{g} / \mathrm{mL})$. After 7-10 days, clusters of transduced cells were observed and re-suspended for future applications. The transduction efficiency of BMSCs in vitro was tested by western blotting.

\section{Rat model of LPS-induced ALI and treatment}

A total of 48 male Sprague-Dawley rats, each weighing approximately 180-200 g, were equally randomized to four groups. Rats were intratracheally instilled with $2 \mathrm{mg} / \mathrm{kg}$ LPS dissolved in $100 \mu \mathrm{L}$ physiological saline. Rats instilled with $100 \mu \mathrm{L}$ of physiological saline were used as controls. Physiological saline, $1 \times 10^{6} \mathrm{BMSC}$ transduced with lentivirus-enhanced green fluorescent protein (eGFP) empty vector $\left(\mathrm{BMSCs}^{\mathrm{ctrl}}\right.$ ) in $200 \mu \mathrm{L}$ saline, or $1 \times 10^{6}$ BMSCs transduced with lentivirus-eGFP NM-II siRNA (BMSCs $^{\text {NM-II KD }}$ ) in $200 \mu \mathrm{L}$ saline were infused via the tail vein 30 min after LPS instillation. Rats from each group were euthanized and lung tissues were collected for further analysis at certain days after treatment.

\section{Histological assessment}

The lung samples were fixed with $4 \%$ formalin, washed, dehydrated, embedded in paraffin, cut into $4 \mu \mathrm{m}$ thick sections, and stained with hematoxylin and eosin (HE) or Masson-Goldner trichrome. Lung injury was scored in a blinded fashion as described previously (15). The degree of lung fibrosis was graded as follows: $0=$ no evidence of fibrosis; $1=$ mild fibrosis; $2=$ moderate fibrosis; and $3=$ severe fibrosis (2).

\section{MPO activity assay}

Lung tissues were homogenized and MPO activity was measured using the corresponding assay kit according to the manufacturer's instructions.

\section{Cell counting and ELISA of IL-1, IL-6, and TNF- $\alpha$ in bronchoalveolar lavage (BAL) fluids}

Postmortem BAL fluids were collected by instilling and withdrawing physiological saline through a tracheal cannula using a catheter. This procedure was repeated three times, and the three BAL fluid samples were pooled and centrifuged. The supernatant portions were stored at $-80{ }^{\circ} \mathrm{C}$ until further examination. Erythrocytes in the cell pellets were lysed using red blood cell lysis buffer and the remaining cells were resuspended in PBS for total cell counting using a hemocytometer. The levels of cytokines IL-1, IL- 6 , and TNF- $\alpha$ in the BAL fluids were measured using ELISA kits according to the manufacturer's instructions.

\section{Quantitative Real-Time PCR (RT-qPCR)}

TRIzol reagent (RT; Qiagen) was used to extract total RNA from lung tissues. RNA was reverse transcribed and amplified using the CFX Connect ${ }^{\mathrm{TM}}$ RT-PCR Detection System (Bio-Rad Laboratories, CA, USA). The primer sequences were as follows: IL- $1 \beta$ [forward primer (FP): 5'-GGGATGATGACGACCTGCTA-3'; reverse primer (RP): 5'-CCACTTGTTGGCTTATGTTCTGT-3'], IL-6 (FP: 5'-TCCTTCCTACCCCAACTTCC-3'; RP: 5'-GGTCCTTAGCCACTCCTTCTG-3'), TNF- $\alpha$ (FP: 5'-CTTCTCATTCCTGCTCGTGG-3'; RP: 5'-CCGCTTGGTGGTTTGCTAC-3'), and GAPDH (FP: 5'-CAACGGGAAACCCATCACCA-3'; RP: 5'-ACGCCAGTAGACTCCACGACAT-3'). 


\section{Cell viability}

Cells were plated in 96-well culture plates and cell viability at certain times was determined using the CCK8 kit (Dojindo Laboratories, Japan) according to the manufacturer's protocol.

\section{Western blot analysis}

Lung tissues or cells were lysed in lysis buffer. Protein concentrations were quantified using a bicinchoninic acid (BCA) protein assay kit (Thermo Scientific, Rockford, IL, USA). Proteins $(60 \mu \mathrm{g})$ were separated by $10 \%$ SDSpolyacrylamide gel electrophoresis and transferred onto nitrocellulose membranes (Millipore, Bedford, MA, USA). After blocking, the membranes were incubated with specific primary antibodies at $4{ }^{\circ} \mathrm{C}$ overnight. The membranes were then washed and incubated with horseradish peroxidaselabeled secondary antibodies. Signals were revealed using the Pierce ECL Plus Western Blotting Substrate (Thermo Scientific, Rockford, IL, USA).

\section{Fluorescence in situ bybridization (FISH)}

Tissue sections were mounted on charged glass slides, deparaffinized, and digested using standard protocol for FISH. Cells were washed three times with precooled PBS and fixed with $4 \%$ paraformaldehyde for $10 \mathrm{~min}$ at room temperature. The probe was labeled with digoxigenin to detect gene expression. The probe was hybridized with tissues at $42{ }^{\circ} \mathrm{C}$ in the dark overnight. The next day, tissue sections were washed five times with saline-sodium citrate buffer at various concentrations, followed by incubation with biotin-conjugated anti-Digoxin and streptavidinFITC (SABC-FITC) (1:100), and counterstaining with 4',6-diamidino-2-phenylindole (DAPI)-antifade solution.

\section{Statistical analysis}

Statistical analyses were performed using SPSS 12.0 (IBM, Armonk, NY, USA). Data are presented as means \pm standard error (SE). Student's $t$-test was used for comparisons between two groups. For comparisons among multiple groups, one-way analysis of variance (ANOVA) was used. Results with $\mathrm{P}<0.05$ were considered statistically significant.

\section{Results}

\section{Phenotype verification of primary BMSCs and NM-II knockdown}

The BMSCs used in the current study were isolated from rats and identified by flow cytometry (Figure 1). BMSCs were negative for CD34/CD44/CD45 and positive for CD29/CD90 mesenchymal markers. The isolated BMSCs were transfected with either NM-II-targeted siRNA or empty vector, and the transduction efficiency was assessed. Western blotting showed that lentivirus-mediated NM-II siRNA efficiently inhibited NM-II expression in BMSCs (Figure 2). Moreover, the stem-cell characteristics of NMII siRNA-modified BMSCs were not significantly changed (data not shown).

\section{Effects of BMSCs ${ }^{\text {NM-II KD }}$ on LPS-induced lung inflammation and pulmonary fibrosis}

Histological examination showed that the intratracheal instillation of LPS could induce significant lung inflammation and pulmonary fibrosis. As shown in Figure 3, LPS treatment produced alveolar wall thickening, neutrophil infiltration, and occasional alveolar hemorrhage. The inflammatory histologic changes produced by LPS in lung parenchyma were significantly attenuated after treatment with either BMSCs ${ }^{c t r l}$ or BMSCs ${ }^{N M-I I ~ K D}$. A significant amelioration in histopathologic changes was achieved by treatment with BMSCs ${ }^{N M-I I}$ KD relative to those seen after BMSCs ${ }^{c t r l}$ treatment. Consistent with this, LPS-induced increases in lung tissue MPO activity and inflammatory cytokine (IL-1, IL-6 and TNF- $\alpha$ ) gene expression were significantly reduced by treatment with BMSC $^{\text {ctrl }}$ and BMSC ${ }^{\text {NM-II KD }}$ (Figure 4A,B). Similarly, MPO activity and mRNA expression of inflammatory cytokines (IL-6 and TNF- $\alpha$ ) were significantly reduced in LPStreated rats that received BMSCS ${ }^{N M-I I} K D$ relative to those detected in rats treated with BMSCs ${ }^{c t r l}$. We also observed that treatment with either $\mathrm{BMSC}^{\mathrm{ctrl}}$ or $\mathrm{BMSC}^{\text {NM-II KD}}$ significantly attenuated LPS-induced inflammation with a decreased concentration of inflammatory cytokines and total cells in BAL fluids (Figure 4C,D). As shown in Figure 5, LPS-induced increases in collagen deposition on day 21, as observed by Masson-Goldner trichrome staining of lung tissues. Both BMSC ${ }^{c t r l}$ and BMSC ${ }^{N M-I I ~ K D}$ treatments markedly reduced the collagen deposition observed in LPS- 
A

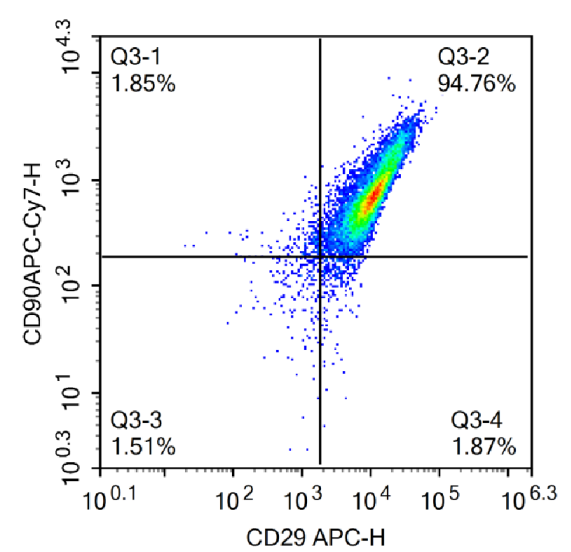

C

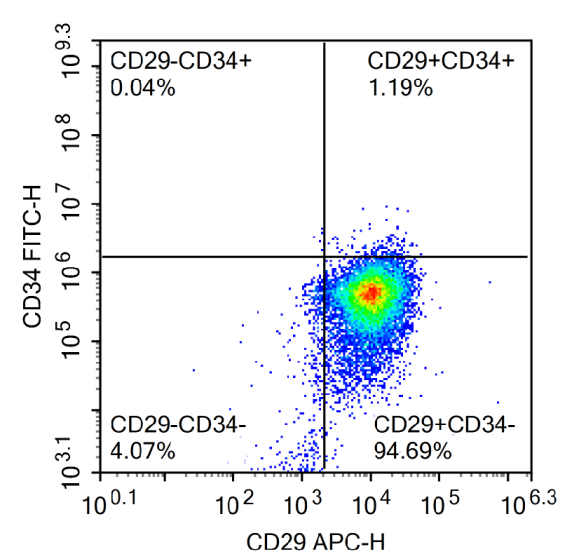

B
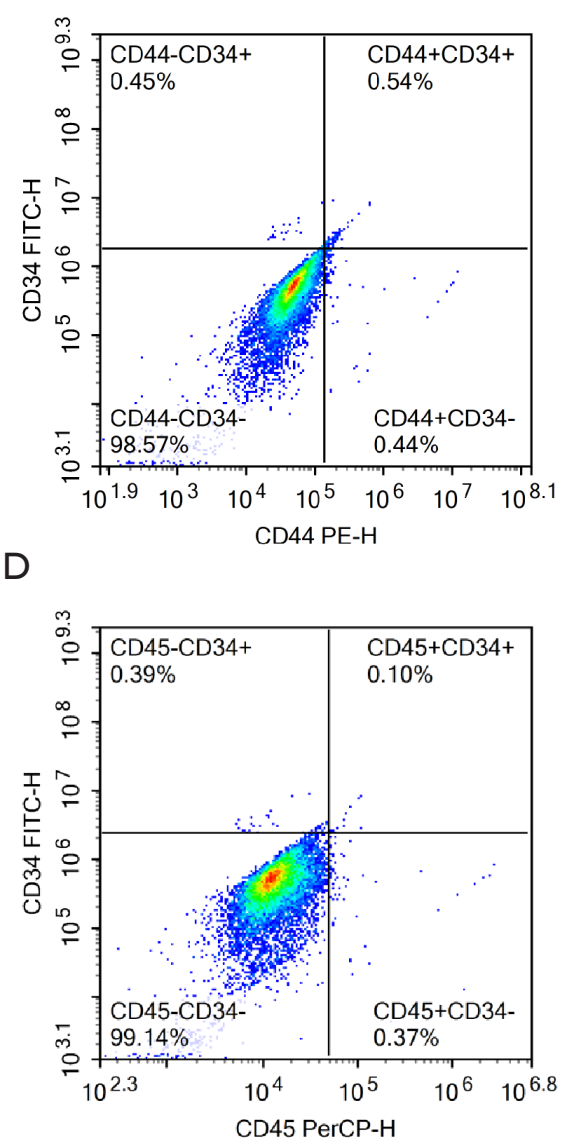

Figure 1 Phenotype verification of primary BMSCs by flow cytometry for expression of multiple CD antigens. BMSC, bone marrowderived mesenchymal stem cell.

A

NM-II

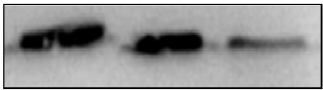

GAPDH

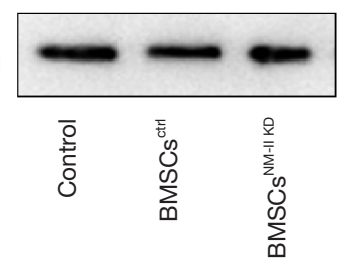

B

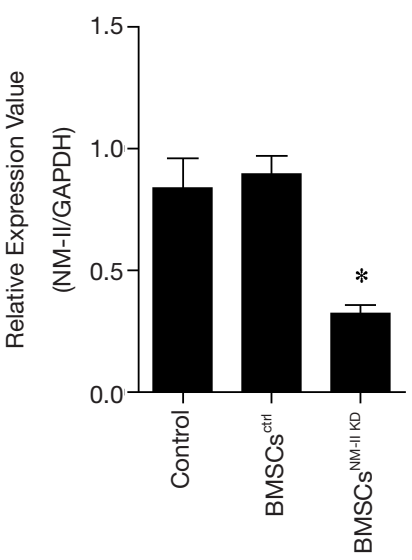

Figure 2 Assessment of transduction efficiency by detecting NM-II expression with western blotting. Representative of three independent experiments ( ${ }^{*}, \mathrm{P}<0.05$, vs. BMSCs $\left.{ }^{\text {crll }}\right)$. BMSC, bone marrow-derived mesenchymal stem cell. 
ALI

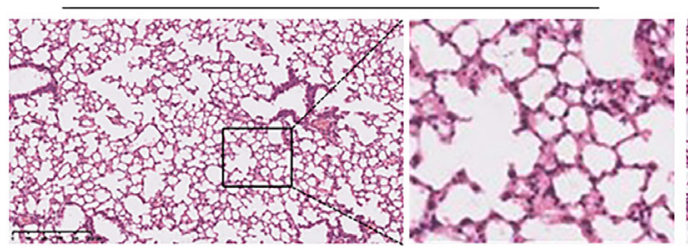

$\mathrm{BMSCs}^{\mathrm{ctrl}}$

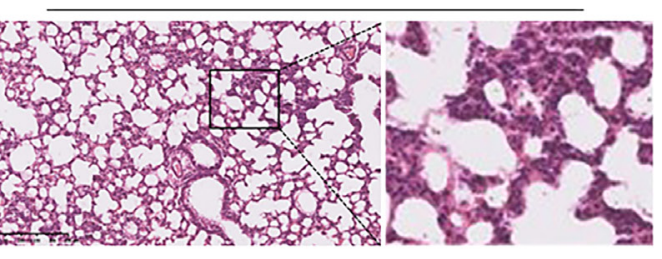

$\mathrm{BMSCs}^{\mathrm{NM}-\mathrm{II} \mathrm{KD}}$

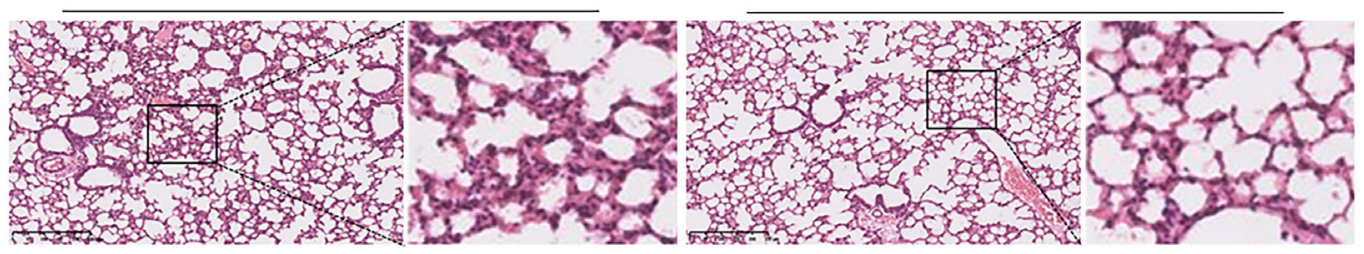

B

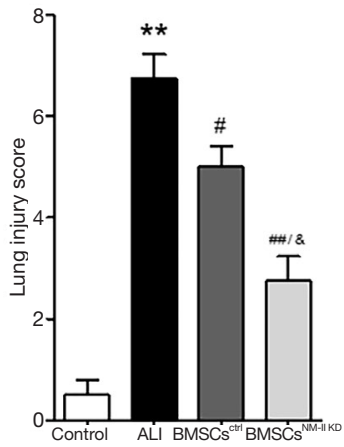

Figure 3 LPS treatment produced alveolar wall thickening, neutrophil infiltration, and occasional alveolar hemorrhage. The inflammatory histologic changes produced by LPS in lung parenchyma were significantly attenuated after treatment with either BMSC ${ }^{\text {ctrl }}$ or BMSCs $^{\text {NM-II }}$ ${ }^{K D}$. A significant amelioration in histopathologic changes was achieved by treatment with BMSCs ${ }^{N M-I I K D}$ relative to those seen after BMSCs ${ }^{c t r l}$ treatment. Histopathological features were tested by hematoxylin and eosin staining on day 1 after treatment (A). Pathological score was measured (B). Data are presented as the mean \pm SE. $\mathrm{n}=4$ in each group. ${ }^{* *}, \mathrm{P}<0.01$ vs. control; ${ }^{*}, \mathrm{P}<0.05,{ }^{\# \#}, \mathrm{P}<0.01$ vs. ALI; ${ }^{\circledR}, \mathrm{P}<0.05$ vs. $\mathrm{BMSCs}^{\text {trtl }}$. ALI, acute lung injury; BMSC, bone marrow-derived mesenchymal stem cell.

A

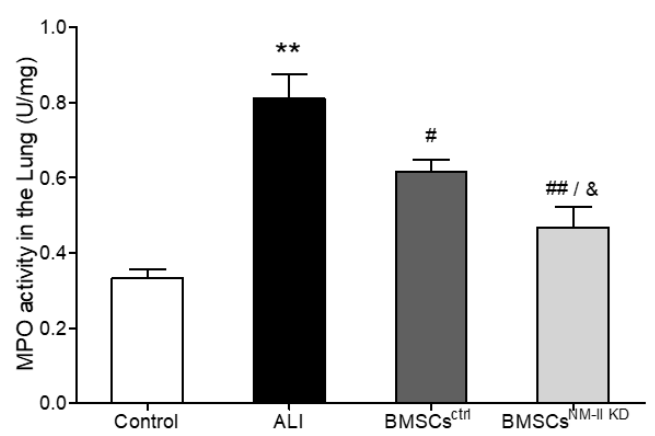

C

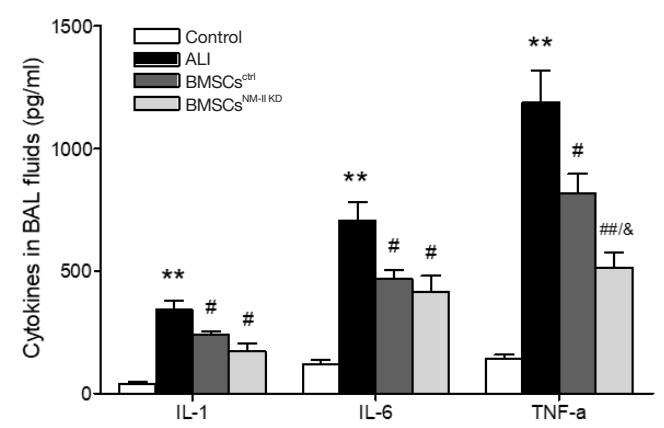

B

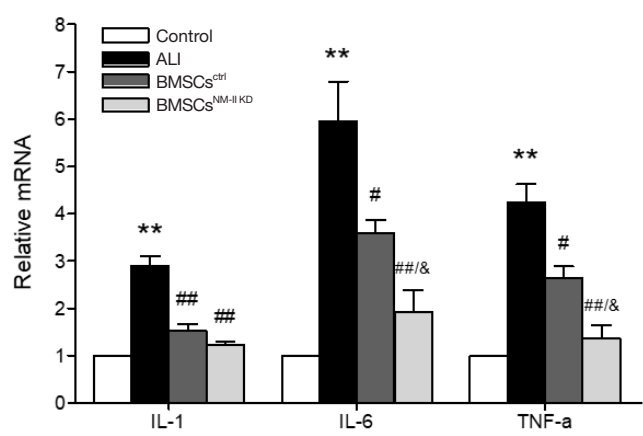

D

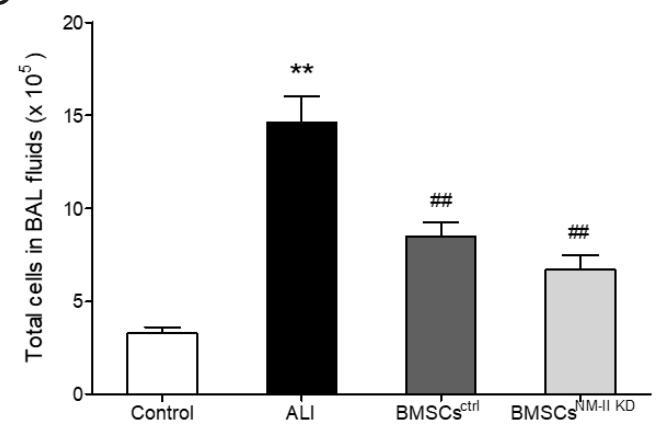

Figure 4 MPO activity (A), mRNA expression of inflammatory cytokines (B), concentrations of inflammatory cytokines in BAL fluids (C) and total cells counts in BAL fluids (D) were measured on day 1 after treatment. Data are presented as the mean $\pm \mathrm{SE} . \mathrm{n}=4$ in each group. ${ }^{* *}$, $\mathrm{P}<0.01$ vs. control; ${ }^{\#}, \mathrm{P}<0.05,{ }^{\text {\#\# }}, \mathrm{P}<0.01$ vs. ALI; ${ }^{\circ}, \mathrm{P}<0.05$ vs. BMSCs ${ }^{\text {crl }}$. MPO, myeloperoxidase; BAL, bronchoalveolar lavage; BMSC, bone marrow-derived mesenchymal stem cell. 
A

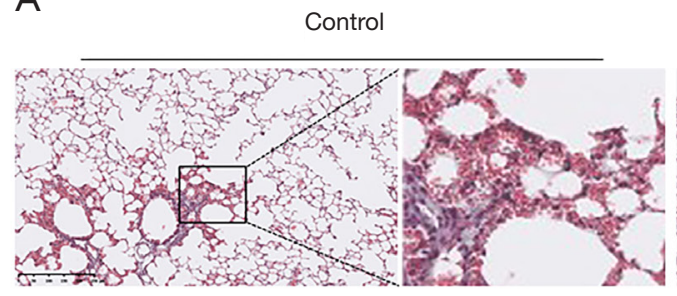

$\mathrm{BMSCs}^{\mathrm{ctrl}}$

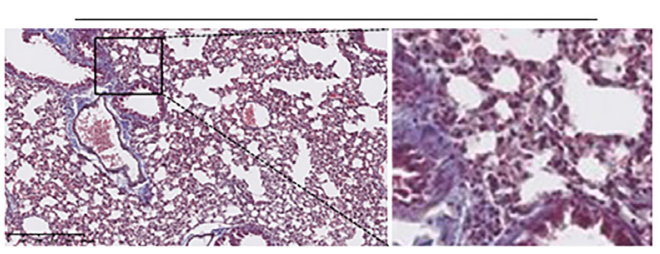

$\mathrm{BMSCs}^{\mathrm{NM}-I I \mathrm{KD}}$

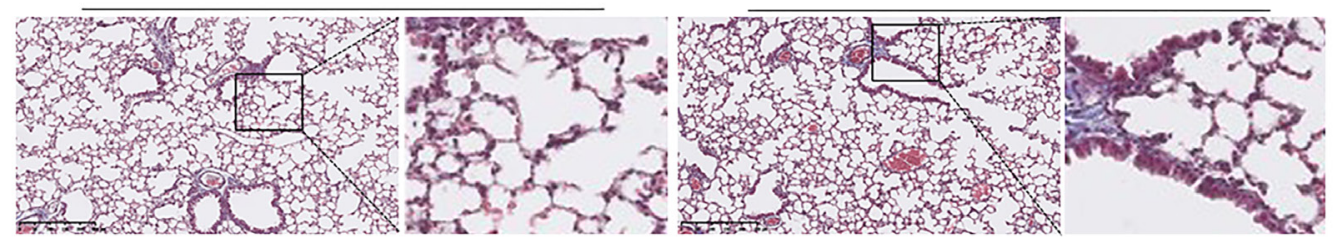

B

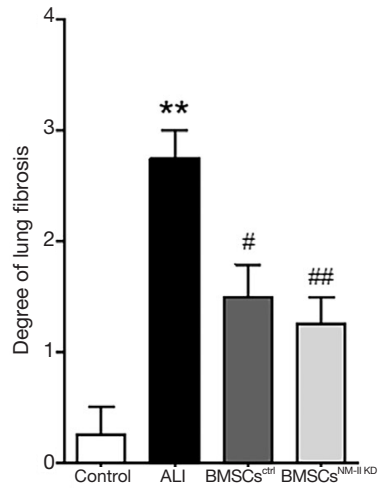

Figure 5 Both BMSCs ${ }^{c t r l}$ and BMSCs ${ }^{N M-I K D}$ treatments markedly reduced the collagen deposition observed in LPS exposed rats. Lung fibrosis was measured at 21 days after treatment by Masson trichrome staining (A). The degree of lung fibrosis was scored (B). Data are presented as the mean \pm SE. $\mathrm{n}=4$ in each group. ${ }^{* *}, \mathrm{P}<0.01$ vs. control; ${ }^{*}, \mathrm{P}<0.05,{ }^{\prime \prime}, \mathrm{P}<0.01$ vs. ALI.

exposed rats.

\section{Survival rate of implanted BMSCs ${ }^{N M-I K D}$ in lung tissues}

FISH, a molecular cytogenetic technique, is used to detect and localize the presence or absence of specific DNA sequences on chromosomes (16). The $S R Y$ gene is a specific gene fragment on the $\mathrm{Y}$ chromosome. Interestingly, the expression level of $S R Y$ can indirectly reflect the number of implanted MSCs. To determine whether silencing of NM-II by RNA interference could affect the survival of BMSCs in injured lung tissues, $S R Y$ gene expression in lung tissues was measured by FISH (Figure 6). On day 1 after intravenous injection of BMSCs, the expression of $S R Y$ was higher in $\mathrm{BMSC}^{\mathrm{ctrl}-}$-and $\mathrm{BMSC}^{\mathrm{NM-IIKD}}$-treated groups compared with that in the control and model groups. Similar results were observed on days 7 and 14 after BMSC treatment. Further analysis indicated that the expression of SRY in the $\mathrm{BMSC}^{\text {NM-II KD }}$-treated group was slightly higher than that in the BMSC ${ }^{\mathrm{ctrl}}$-treated group. These results suggested that silencing NM-II in BMSCs might enhance cellular survival in this LPS-induced ALI model, and that BMSCs could last as long as 2 weeks post-transfection.

\section{Effect of NM-II-targeted siRNA transfection on self-renewal activity of BMSCs and apoptosis-associated protein expression}

As shown in Figure $7 A$, results revealed that NM-II knockdown inhibited the decreased of BMSCs viability; even slightly higher inhibition was observed at 96h. Nanog and Ang-1 expression indicate the self-renewal activity of stem cells. Therefore, we examined Nanog and Ang-1 levels by western blotting. As shown in Figure 7B, Nanog and Ang-1 were significantly upregulated in BMSCs upon NMII knockdown, indicating that endogenous knockdown of NM-II may enhance the self-renewal capacity of implanted BMSCs. Interestingly, the activity of Caspase-3, a marker of cell apoptosis, was decreased in BMSCs upon NM-II knockdown.

\section{Effect of NM-II knockdown on Rho/ROCK activity}

The Rho/ROCK signaling pathway plays important roles in mediating cell fate toward self-renewal or apoptosis. As shown in Figure 7B,C, NM-II knockdown significantly decreased the expression of ROCK1 and ROCK2 in BMSCs, indicating that downregulation of NM-II could reduce ROCK activity, thus inhibiting the apoptosis of BMSCs and promoting self-renewal.

\section{Discussion}

Endotoxin-induced lung injury is characterized by significant inflammatory damage and edema at early stages. In addition, endotoxin exposure can stimulate fibrotic tissue formation at later stages (17). The present study, together with some previous reports $(18,19)$, showed that intravenous administration of BMSCs and NM-II knockdown of 

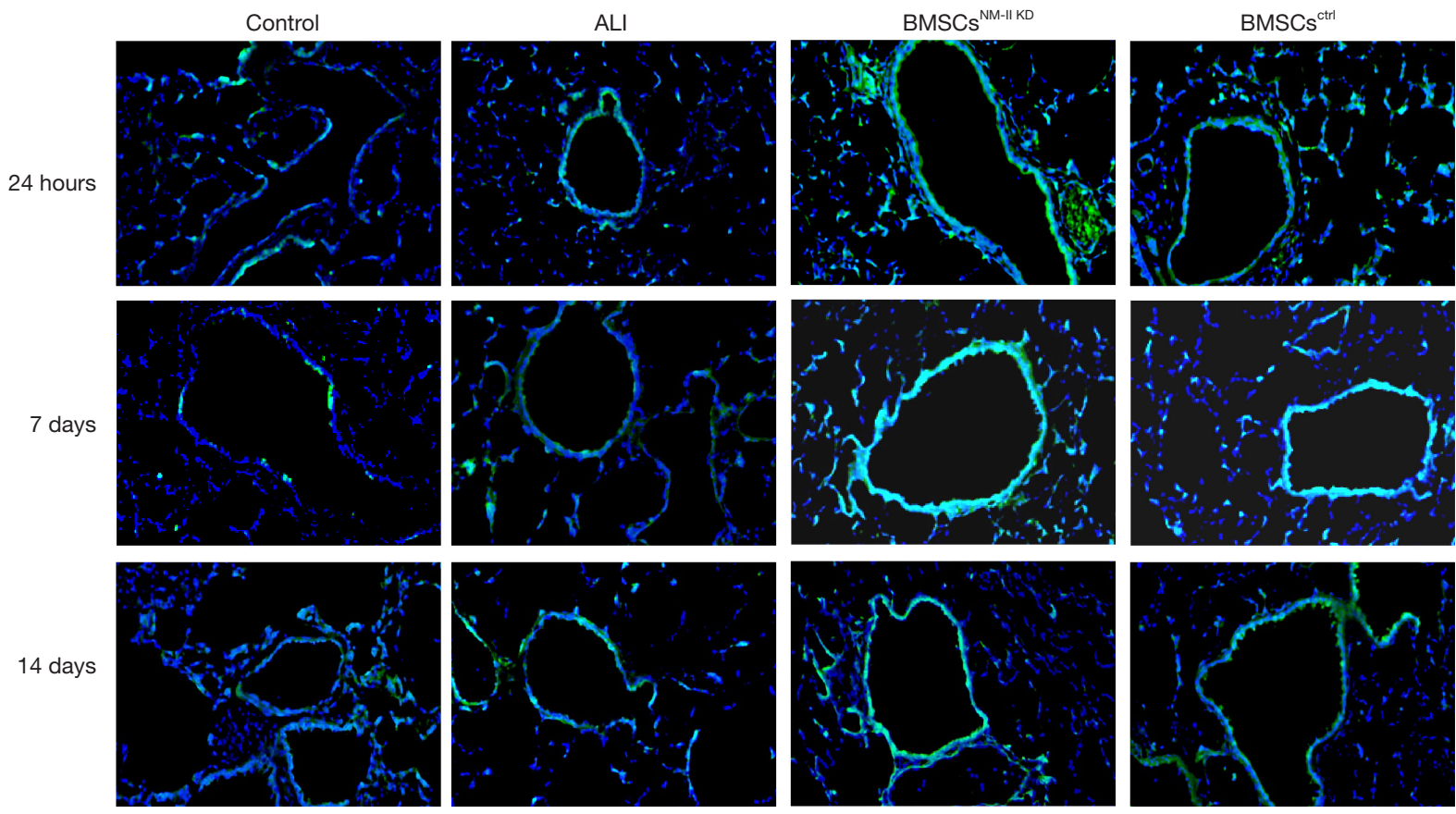

Figure 6 SRY (sex-determining region of the Y chromosome) gene expression was measured by FISH at 24 hours, 7 days and 14 days after treatment.

A

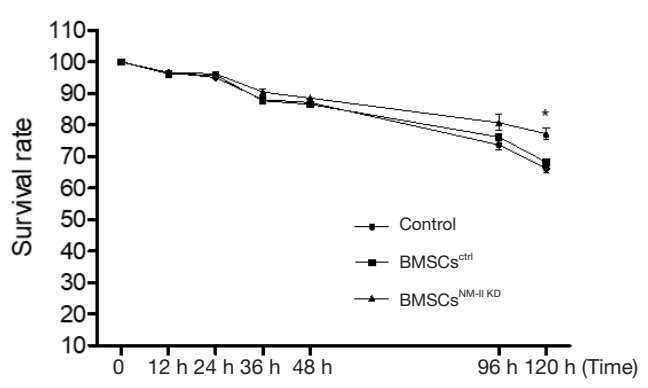

C

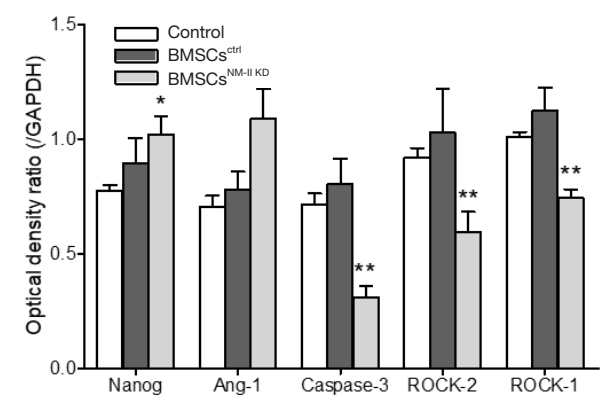

B

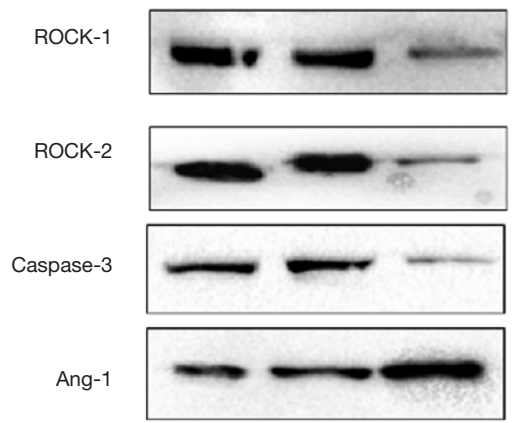

Nanog

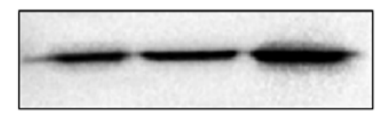

GAPDH

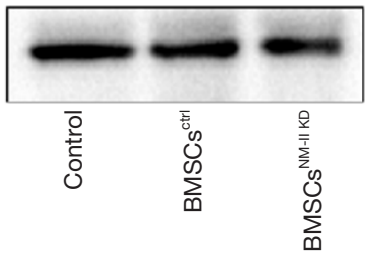

Figure 7 NM-II knockdown inhibited the decreased of BMSCs viability. Nanog and Ang-1 were significantly upregulated in BMSCs upon NM-II knockdown, while the activity of Caspase-3 was decreased in BMSCs upon NM-II knockdown. Cell viability was measured by CCK8 assay (A). Expression of self-renewal and apoptosis-associated proteins were evaluated by western blotting (B). Representative of three independent experiments. Relative protein levels were quantified by densitometry and expressed as optical density ratio with GAPDH serving as internal standards $(\mathrm{C})$. ${ }^{*}, \mathrm{P}<0.05,{ }^{* *}, \mathrm{P}<0.01$ vs. control. 
BMSCs improved histopathological morphology, decreased inflammatory infiltrates, downregulated the expression levels of proinflammatory cytokines, and reduced pulmonary interstitial edema. Furthermore, treatment with both BMSCs and NM-II siRNA-modified BMSCs inhibited fibrosis and alleviated the degree of endotoxininduced pulmonary fibrosis. We also found that NM-II knockdown in BMSCs showed a slightly better therapeutic effect on lung inflammation than control BMSCs. However, in the present study, we did not examine any physiological parameter, such as lung function or $\mathrm{O}_{2}$ saturation in the acute phase, since in our clinical practice we have observed that stem cells are less likely to improve such parameters. These encouraging results would not be suitable for direct application in patients with ALI at this moment. Further research is needed for the conversion from basic to clinical applications.

In addition to inflammatory responses, pathological changes in ALI involve hypoxia and free radical damage. It has been reported that ischemia, oxidative stress, and an inflammatory microenvironment might contribute to necrosis or apoptosis of transplanted stem cells, leading to low transplantation and survival rates (10,20-22). In the present study, we demonstrated that most BMSCs underwent apoptosis or disappeared a few days after transplantation into lung tissues.

Recently, various strategies such as statin treatment, ischemia preconditioning, and genetic modification have been used to increase the survival ratio of MSCs, and improve therapeutic efficiency after implantation (10,14,23-25). $\mathrm{Li}$ et al. (14) confirmed that Bcl-2 overexpression could inhibit MSC death and apoptosis under hypoxic conditions, and genetic modification of MSCs with Bcl-2 could play significant roles in improving the efficacy of stem cell therapy. These findings suggest that regulating MSC apoptosis may be useful for improving cell viability. In the present study, we demonstrated that BMSCs could be transfected with NM-II siRNA with high efficiency. The expression of self-renewal-associated proteins, such as Nanog and Ang-1, was upregulated in NM-II knockdown BMSCs, which contributed to the self-renewal of stem cells. Our results were consistent with previous findings from Walker et al. (13) who showed that treatment with NM-II inhibitors could enhance stem cell survival. We also demonstrated that transfection with NM-II-targeted siRNA decreased caspase-3 activity, suggesting that NMII knockdown could protect implanted BMSCs against apoptosis.
ROCK signaling is one of the key regulators of cytoskeletal dynamics during the cell cycle and thus helps to maintain the balance between stem cell self-renewal and apoptosis (26). It was previously shown that treatment with the ROCK inhibitor Y-27632 can efficiently enhance stem cell survival (27). A previous study reported that ROCK serves as a master regulator of NM-II activity (28). However, in this study, we found that NM-II knockdown led to reduced ROCK activity. Therefore, we hypothesize that downregulation of NM-II gene expression in BMSCs could reduce ROCK activity, thus inhibiting BMSCs apoptosis and promoting self-renewal. The precise mechanisms underlying these phenomena need to be further investigated.

\section{Conclusions}

In summary, NM-II knockdown in BMSCs provided a slightly enhanced ability to attenuate lung injury after LPS challenge. These benefits might be partially associated with decreased apoptosis and improved self-renewal in NMII siRNA-modified BMSCs. These findings provide novel insights for developing effective approaches for treating LPS-induced ALI in the future.

\section{Acknowledgments}

Funding: This work was supported by National Natural Science Foundation of China $(81801937,81772125$, 81701899).

\section{Footnote}

Reporting Checklist: The authors have completed the ARRIVE reporting checklist. Available at http://dx.doi. org/10.21037/atm-20-4851

Data Sharing Statement: Available at http://dx.doi. org/10.21037/atm-20-4851

Conflicts of Interest: All authors have completed the ICMJE uniform disclosure form (available at http://dx.doi. org/10.21037/atm-20-4851). The authors have no conflicts of interest to declare.

Ethical Statement: The authors are accountable for all aspects of the work in ensuring that questions related to the accuracy or integrity of any part of the work 
are appropriately investigated and resolved. This work has been approved by the Animal Care and Use Committee of the Naval Medical University (Approval ID: SYXK(HU)2017-0004), in compliance with guide for care and use of laboratory animals published by the U.S. National Institutes of Health (NIH) (publication No. 96-01).

Open Access Statement: This is an Open Access article distributed in accordance with the Creative Commons Attribution-NonCommercial-NoDerivs 4.0 International License (CC BY-NC-ND 4.0), which permits the noncommercial replication and distribution of the article with the strict proviso that no changes or edits are made and the original work is properly cited (including links to both the formal publication through the relevant DOI and the license). See: https://creativecommons.org/licenses/by-nc-nd/4.0/.

\section{References}

1. Mohammadipoor A, Antebi B, Batchinsky AI, et al. Therapeutic potential of products derived from mesenchymal stem/stromal cells in pulmonary disease. Respir Res 2018;19:218.

2. Cui P, Xin H, Yao Y, et al. Human amnion-derived mesenchymal stem cells alleviate lung injury induced by white smoke inhalation in rats. Stem Cell Res Ther 2018;9:101.

3. Islam MN, Das SR, Emin MT, et al. Mitochondrial transfer from bone-marrow-derived stromal cells to pulmonary alveoli protects against acute lung injury. Nat Med 2012;18:759-65.

4. Ortiz LA, Gambelli F, McBride C, et al. Mesenchymal stem cell engraftment in lung is enhanced in response to bleomycin exposure and ameliorates its fibrotic effects. Proc Natl Acad Sci U S A 2003;100:8407-11.

5. Zhu F, Wang J, Qiu X, et al. Smoke inhalation injury repaired by a bone marrow-derived mesenchymal stem cell paracrine mechanism: Angiogenesis involving the Notch signaling pathway. J Trauma Acute Care Surg 2015;78:565-72.

6. Zhu F, Xia ZF. Paracrine activity of stem cells in therapy for acute lung injury and adult respiratory distress syndrome. J Trauma Acute Care Surg 2013;74:1351-6.

7. Laffey JG, Matthay MA. Fifty Years of Research in ARDS. Cell-based Therapy for Acute Respiratory Distress Syndrome. Biology and Potential Therapeutic Value. Am J Respir Crit Care Med 2017;196:266-73.

8. Wilson JG, Liu KD, Zhuo H, et al. Mesenchymal stem (stromal) cells for treatment of ARDS: a phase 1 clinical trial. Lancet Respir Med 2015;3:24-32.

9. Zheng G, Huang L, Tong H, et al. Treatment of acute respiratory distress syndrome with allogeneic adiposederived mesenchymal stem cells: a randomized, placebocontrolled pilot study. Respir Res 2014;15:39.

10. Chen S, Chen $\mathrm{L}, \mathrm{Wu} X$, et al. Ischemia postconditioning and mesenchymal stem cells engraftment synergistically attenuate ischemia reperfusion-induced lung injury in rats. J Surg Res 2012;178:81-91.

11. Hoffman AM, Paxson JA, Mazan MR, et al. Lung-derived mesenchymal stromal cell post-transplantation survival, persistence, paracrine expression, and repair of elastaseinjured lung. Stem Cells Dev 2011;20:1779-92.

12. Vicente-Manzanares M, Ma X, Adelstein RS, et al. Nonmuscle myosin II takes centre stage in cell adhesion and migration. Nat Rev Mol Cell Biol 2009;10:778-90.

13. Walker A, Su H, Conti MA, et al. Non-muscle myosin II regulates survival threshold of pluripotent stem cells. Nat Commun 2010;1:71.

14. Li W, Ma N, Ong LL, et al. Bcl-2 engineered MSCs inhibited apoptosis and improved heart function. Stem cells 2007;25:2118-27.

15. Sun Y, Qiu X, Wu G, et al. The effects of porcine pulmonary surfactant on smoke inhalation injury. J Surg Res 2015;198:200-7.

16. Levsky JM, Singer RH. Fluorescence in situ hybridization: past, present and future. J Cell Sci 2003;116:2833-8.

17. Suzuki T, Tada Y, Gladson S, et al. Vildagliptin ameliorates pulmonary fibrosis in lipopolysaccharide-induced lung injury by inhibiting endothelial-to-mesenchymal transition. Respir Res 2017;18:177.

18. Chen J, Shao Y, Xu G, et al. Bone marrow-derived mesenchymal stem cells attenuate phosgene-induced acute lung injury in rats. Inhal Toxicol 2015;27:254-61.

19. Zheng Y, Cai W, Zhou S, et al. Protective effect of bone marrow derived mesenchymal stem cells in lipopolysaccharide-induced acute lung injury mediated by claudin-4 in a rat model. Am J Transl Res 2016;8:3769-79.

20. Zhang M, Methot D, Poppa V, et al. Cardiomyocyte grafting for cardiac repair: graft cell death and anti-death strategies. J Mol Cell Cardiol 2001;33:907-21.

21. Balsam LB, Wagers AJ, Christensen JL, et al. Haematopoietic stem cells adopt mature haematopoietic fates in ischaemic myocardium. Nature 2004;428:668-73.

22. Deng J, Yang C, Wang Y, et al. Inositol pyrophosphates mediated the apoptosis induced by hypoxic injury in bone marrow-derived mesenchymal stem cells by autophagy. Stem Cell Res Ther 2019;10:159. 
23. Yang YJ, Qian HY, Huang J, et al. Atorvastatin treatment improves survival and effects of implanted mesenchymal stem cells in post-infarct swine hearts. Eur Heart J 2008;29:1578-90.

24. Tsubokawa T, Yagi K, Nakanishi C, et al. Impact of antiapoptotic and anti-oxidative effects of bone marrow mesenchymal stem cells with transient overexpression of heme oxygenase-1 on myocardial ischemia. Am J Physiol Heart Circ Physiol 2010;298:H1320-9.

25. Dong Q, Yang Y, Song L, et al. Atorvastatin prevents mesenchymal stem cells from hypoxia and serum-free injury through activating AMP-activated protein kinase.

Cite this article as: $\mathrm{Wu} G$, Chang F, Fang H, Zheng $\mathrm{X}$, Zhuang M, Liu X, Hou W, Xu L, Chen Z, Tang C, Wu Y, Sun Y, Zhu F. Non-muscle myosin II knockdown improves survival and therapeutic effects of implanted bone marrowderived mesenchymal stem cells in lipopolysaccharide-induced acute lung injury. Ann Transl Med 2021;9(3):262. doi: 10.21037/ atm-20-4851
Int J Cardiol 2011;153:311-6.

26. Liu $X$, Zhang Z, Yan X, et al. The Rho kinase inhibitor Y-27632 facilitates the differentiation of bone marrow mesenchymal stem cells. J Mol Histol 2014;45:707-14.

27. Heng BC. Effect of Rho-associated kinase (ROCK) inhibitor Y-27632 on the post-thaw viability of cryopreserved human bone marrow-derived mesenchymal stem cells. Tissue cell 2009;41:376-80.

28. Newell-Litwa KA, Horwitz R, Lamers ML. Nonmuscle myosin II in disease: mechanisms and therapeutic opportunities. Dis Model Mech 2015;8:1495-515. 